\title{
Primary Sjögren's syndrome preceding the presentation of systemic lupus erythematosus as a benign intracranial hypertension syndrome
}

\author{
X Chevalier, M de Bandt, P Bourgeois, M F Kahn
}

\author{
Rheumatology \\ Department, \\ Hôpital Henri-Mondor, \\ Creteil, France \\ $\mathrm{X}$ Chevalier \\ Rheumatology \\ Department, \\ Hôpital Bichat, Paris, \\ France \\ $M$ de Bandt \\ Rheumatology \\ Department, \\ Université Paris VI, \\ Hôpital de la Pitié, \\ Paris, France \\ P Bourgeois \\ Rheumatology \\ Department, \\ Université Paris VII, \\ Hôpital Bichat, Paris, \\ France \\ M F Khan \\ Correspondence to: \\ Dr X Chevalier, \\ Boulevard de Lattre \\ de Tassigny, 94010 Creteil, \\ France. \\ Accepted for publication \\ 30 July 1991
}

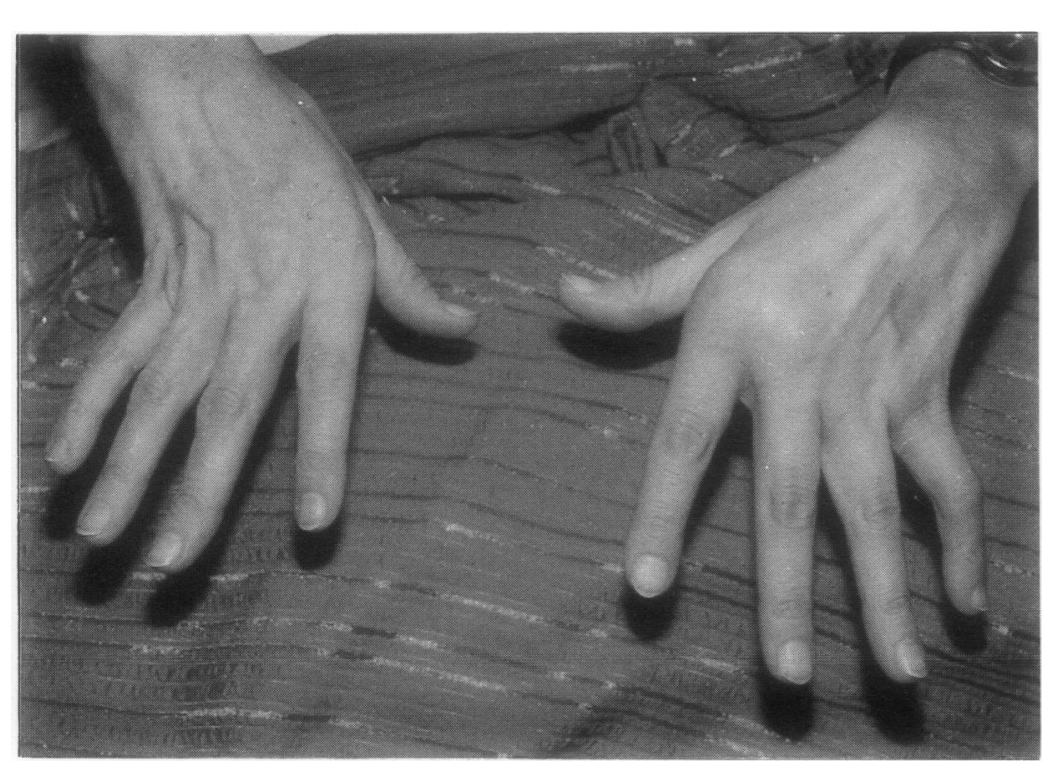

Deformation of the patient's hands showing an ulna deviation typical of faccoud's arthritis.

\begin{abstract}
The case is reported of a 41 year old white woman who developed systemic lupus erythematosus (SLE) seven years after primary Sjögren's syndrome and four years after the association of Sjögren's syndrome with Jaccoud's arthritis. The SLE was detected by a benign intracranial hypertension, which is a rare neuro-ophthalmic presentation of lupus. No associated conditions linked to benign intracranial hypertension syndrome were seen in this patient and the hypertension syndrome recurred one year later.
\end{abstract}

Systemic lupus erythematosus (SLE) may be associated with Sjögren's syndrome. ${ }^{1}$ In addition, some patients with longstanding Sjögren's syndrome may develop systemic manifestations fulfilling the American Rheumatism Association 1982 criteria for SLE. ${ }^{2}$ We report here the case of a 41 year old woman with Sjögren's syndrome who developed symptoms of benign intracranial hypertension syndrome leading to a secondary diagnosis of SLE. Benign intracranial hypertension syndrome consists of increased intracranial pressure without an expanding mass or hydrocephalus. ${ }^{3}$ This neuro-ophthalmic manifestation is a rare event in the course of SLE. ${ }^{4}$ The mechanism of this increased intracranial pressure is not clearly understood. ${ }^{3}$ Some workers have shown the presence of a dural sinus thrombosis associated with benign intracranial hypertension in patients with SLE; this was not present in our patient. ${ }^{5-7}$

Case report

A 41 year old woman presented in 1981 with polyarthralgia associated with Raynaud's phenomenon and sicca symptoms. Joint pain had first begun at the age of 18 years. A diagnosis of primary Sjögren's syndrome was confirmed by a positive Schirmer's test, the absence of articular erosions on radiological examination, hypergammaglobulinaemia $(28 \mathrm{~g} / \mathrm{l})$ (normal $<12 \mathrm{~g} / \mathrm{l}$ ), and grade 3 on Chislom's classification of labial biopsy samples. Serological markers of SLE (antibodies to DNA and antinuclear antibodies) were absent. The patient was then treated with $10 \mathrm{mg}$ prednisone and chloroquine. During the following four years, a progressive hand deformation occurred (fig), consisting of ulna deviation and metacarpophalangeal subluxation without articular erosion on radiological examination. Antinuclear antibodies (on rat liver) were slightly positive at 1/80 with a speckled pattern and antibodies to DNA (determined by radioimmunoassay) were absent.

In June 1988 she reported severe bitemporal headaches with nausea but without fever. She was receiving $10 \mathrm{mg}$ prednisone daily. Her blood pressure and a neurological examination were normal. Ophthalmic examination, however, showed a bilateral papilloedema. The erythrocyte sedimentation rate was slightly increased $(26 \mathrm{~mm} /$ hour ) (normal $<10 \mathrm{~mm} /$ hour). Sideropenic anaemia (haemoglobin $80 \mathrm{~g} / \mathrm{l}$ ) was present partly due to important metrorragias. Other laboratory tests showed lymphopenia (leucocytes $0.76 \times 10^{9} / 1$; normal $1.5-4 \cdot 0 \times 10^{9} / 1$ ), hypergammaglobulinaemia $(26 \mathrm{~g} / \mathrm{l})$, hypocomplementaemia (CH50 56\%), positive titre for antibodies to DNA (40 IU; positivity $>10$ IU), positive antinuclear antibodies (1/100; positivity $>1 / 80$ ), and the presence of antibodies to Ro. There were no antibodies against phospholipids. Coagulation tests were normal. A computed tomographic scan of the head showed no focal lesions but only small ventricles. Lumbar puncture produced a clear cerebral fluid under an opening pressure of $210 \mathrm{~mm}$ $\mathrm{H}_{2} \mathrm{O}$ There were no cells and the protein concentration was $300 \mathrm{mg} / \mathrm{l}$. Digital subtraction angiography showed neither sinus nor venous thrombosis. A diagnosis of benign intracranial hypertension associated with SLE was made. The patient received $30 \mathrm{mg} /$ day prednisone by mouth. Her symptoms dramatically resolved in a few days as did the papilloedema. One year after this episode joint paiil and the benign intracranial hypertension syndrome recurred. Magnetic resonance imaging performed during this second episode showed neither local 
vasculitis nor indirect signs of venous thrombosis. The patient was again successfully treated by high doses of prednisone.

\section{Discussion}

Benign intracranial hypertension syndrome is a syndrome of increased intracranial pressure with papilloedema not associated with focal neurological signs. ${ }^{3}$ Four conditions are required to confirm this syndrome; increased cerebrospinal fluid pressure over $200 \mathrm{~mm} \mathrm{H}_{2} \mathrm{O}$, normal cellular and biochemical composition of the cerebrospinal fluid, symptoms and signs restricted to those of increased intracranial pressure, and normal radiological findings. ${ }^{3}$ In our patient it is unlikely that the benign intracranial hypertension syndrome can be regarded as a manifestation of Sjögren's disease. Indeed, benign intracranial hypertension syndrome has not been reported as a neurological manifestation in Sjögren's syndrome, ${ }^{8}$ although it is a rare event in the course of SLE. Only a few cases have been reported. ${ }^{7-9}$

Benign intracranial hypertension syndrome as a presenting manifestation of SLE is extremely rare. ${ }^{4} 10$ There have been many conditions associated with benign intracranial hypertension syndrome, though most of the cases are idiopathic. Ashlkog and O'Neill found only 11 sufficiently documented conditions in a review of published work: therapeutic drugs including corticosteroids, nalidix acid, nitrofurantoin, tetracycline, and vitamin A; systemic conditions such as anaemia, hypovitaminosis $A$, sarcoidosis and SLE; obesity; and hypertension. " Anaemia and hypertension may occur as a part of SLE itself. $^{59}$ In our patient blood pressure was normal and anaemia was not severe enough to explain the benign intracranial hypertension syndrome. ${ }^{9}$ Corticosteroids may also play a part in SLE associated with benign intracranial hypertension syndrome. In this instance, however, the patient had received $10 \mathrm{mg}$ prednisone without any change in dose before her admission to hospital. More interesting reports show the occurrence of dural sinus thrombosis as a cause of benign intracranial hypertension syndrome in patients with SLE. ${ }^{6}$ An increased risk of thrombosis in SLE may be due to various reasons such as lupus anticoagulant, low levels of antibodies to thrombin III associated with lupus nephritis, or the presence of fibrin split products. ${ }^{12}$ In our patient no abnormality of coagulation tests or cerebral venous thrombosis could be detected. During the recurrent episode of benign intracranial hypertension syndrome, magnetic resonance imaging did not show any abnormalities in signal intensity to suggest vascular injury.

Hence dural sinus thrombosis may be regarded as a potential cause of benign intracranial hypertension syndrome occurring in patients with SLE, but not the only cause. It is difficult to correlate benign intracranial hypertension syndrome as directly related to central nervous system vasculitis except by exclusion. The treatment of benign intracranial hypertension syndrome occurring during the course of SLE requires corticosteroids, which usually lead to a rapid improvement, ${ }^{5} 9$ though persistently increased cerebrospinal fluid pressure despite treatment with corticosteroids can be observed in $10 \%$ of patients with benign intracranial hypertension syndrome. ${ }^{13}$

Another remarkable feature of this patient was the presence of Jaccoud's arthritis early in the course of primary Sjögren's syndrome. Jaccoud's syndrome preceded by four years the diagnosis of SLE and occurred 23 years after the first articular symptoms. The patient had no past medical history of rheumatic fever, another cause of Jaccoud's arthropathy. ${ }^{14}$ Jaccoud's arthritis has been described in patients with lupus with an occurrence of $4-5 \%$ an average of 14 years after onset. ${ }^{14}$ It has been rarely reported in other connective tissue diseases, including primary Sjögren's syndrome, scleroderma, polymyositis, and mixed connective tissue disease. ${ }^{15}$ It has also been mentioned as the most common occurrence of sicca syndrome in patients with SLE and Jaccoud's deformation, an association close to that in our patient.

1 Steinberg A D, Talal N. The coexistence of Siögren's syndrome and systemic lupus erythematosus. Ann Intern Med 1971; 74: 55-61.

2 Tan E M, Cohen A S, Fries J F, et al. The 1982 revised criteria for the classification of systemic lupus erythematosus. Arthritis Rheum 1982; 25: 1271-7.

3 Corbett J J. Problems in the diagnosis and treatment of pseudo-tumor cerebri. Can $\mathcal{7}$ Neurol Sci 1983; 10: 221-9.

4 Feinglass E J, Arnett F C, Dorsh C A, Zizic T D, Stevens M B. Neuropsychiatric manifestations of systemic lupus erythematosus: diagnosis, clinical spectrum and relationship to the others features of the disease. Medicine (Baltimore) 1976; 55: 323-37

$5 \mathrm{Li} \mathrm{E} \mathrm{K}$, Ho P C. Pseudo-tumor cerebri in systemic lupus erythematosus. I Rheumatol 1989; 16: 113-6.

6 Parnass S M, Goodwin J A, Patel D V, Levison D J, D'Arc Rheinhard J. Dural sinus thrombosis: a mechanism for pseudo-tumor cerebri in systemic lupus erythematosus. $f$ Rheumatol 1987; 14: 152-5.

7 Kaplan R E, Springate J E, Feld L G. Pseudo-tumor cerebri associated with cerebral venous sinus thrombosis, internal jugular vein thrombosis and systemic lupus erythematosus. jugular vein thrombosis an

8 Alexander E L, Beail S S, Gordon B, et al. Magnetic cerebral imaging of cerebral lesions in patients with Sjögren's syndrome. Am 7 Med 1988; 85: 513-8.

9 Silberberg D H, Laties A M. Increased intracranial pressure in disseminated lupus erythematosus. Arch Neurol 1973; 29: 88-90.

10 Delgiudice G C, Scher C A, Athrey A B H, Diamond G R. Pseudo-tumor cerebri and childhood systemic lupus erythematosus. F Rheumatol 1986; 13: 748-52.

11 Ashlkog J E, O'Neill B P. Pseudo-tumor cerebri. Ann Intern

Med 1982: 97: 249-56.
12 Mueh J R, Herbst K D, Rapaport J F. Thrombosis in patients with systemic lupus erythematosus. Ann Intern Med 1980; 92: 156-9.

13 Hoffman H J. How is pseudo-tumor cerebri diagnosed? Arch Neurol 1986; 2: 167-8.

14 Bywaters E G L. Jaccoud's syndrome. Clin Rheum Dis 1975; 1: $125-48$.

15 Villiaumey J, Arlet J, Avouac B, et al. Diagnostic criteria and new etiologic events in the arthropathy of Jaccoud: a report of ten cases. Clin Rheumatol 1986; 4: 156-75. 